PROCEEDINGS OF THE

AMERICAN MATHEMATICAL SOCIETY

Volume 137, Number 6, June 2009, Pages 1977-1987

S 0002-9939(09)09773-1

Article electronically published on January 21, 2009

\title{
ON THE EXISTENCE OF INFINITE ENERGY SOLUTIONS FOR NONLINEAR SCHRÖDINGER EQUATIONS
}

\author{
PABLO BRAZ E SILVA, LUCAS C. F. FERREIRA, AND ELDER J. VILLAMIZAR-ROA
}

(Communicated by Hart F. Smith)

\begin{abstract}
We derive new results about existence and uniqueness of local and global solutions for the nonlinear Schrödinger equation, including self-similar solutions. Our analysis is performed in the framework of weak- $L^{p}$ spaces.
\end{abstract}

\section{INTRODUCTION}

We consider the nonlinear Schrödinger equations

$$
\begin{aligned}
i \partial_{t} u+\Delta u & =\lambda|u|^{\rho} u, x \in \mathbb{R}^{n}, t \in \mathbb{R}, \\
u(0, x) & =\phi(x), x \in \mathbb{R}^{n},
\end{aligned}
$$

where $u=u(t, x)$ is a complex-valued function, $\lambda$ is a fixed complex number, and $0<\rho<\infty$. The initial value $\phi: \mathbb{R}^{n} \rightarrow \mathbb{C}$ is given. The Cauchy problem (1.1)-(1.2) is formally equivalent to the integral equation

$$
u(t)=S(t) \phi-i \lambda \int_{0}^{t} S(t-s)\left(|u(s)|^{\rho} u(s)\right) d s,
$$

where $S(t)$ is the unitary group determined by the linear Schrödinger equation

$$
\partial_{t} u-i \Delta u=0, x \in \mathbb{R}^{n}, t \in \mathbb{R} .
$$

If $\phi \in \mathcal{S}\left(\mathbb{R}^{n}\right)$ and $u$ is defined by $\widehat{u(t)}(\xi)=e^{-i|\xi|^{2} t} \widehat{\phi}(\xi)$, for $\xi \in \mathbb{R}^{n}$, then

$$
\widehat{u}_{t}+i|\xi|^{2} \widehat{u}=0
$$

in $\mathbb{R} \times \mathbb{R}^{n}$. In this case, the solution of

$$
\begin{gathered}
\partial_{t} u-i \Delta u=0, x \in \mathbb{R}^{n}, t \in \mathbb{R}, \\
u(0, x)=\phi(x), x \in \mathbb{R}^{n},
\end{gathered}
$$

is given by $u(t)=S(t) \phi=K_{t} * \phi$, where $K_{t}(x)=\left(e^{-i|\xi|^{2} t}\right)$.

The existence and uniqueness of local and global solutions of problem (1.1)-(1.2) have been much studied in the framework of the Sobolev spaces $H^{s}, s \geq 0$; i.e., the solutions and their derivatives have finite energy in the sense that they have finite $L^{2}$-norm. See, for instance, 3, 4, 5, 9, 10, 11, 12, 13, and the references therein.

Received by the editors December 12, 2007.

2000 Mathematics Subject Classification. Primary 35Q55, 35D05, 35B40.

The first author was partly supported during this work by CAPES/MECD-DGU Brazil/Spain, grant No. $117 / 06$. 
As far as we know, the first authors to study infinite energy solutions of (1.1)(1.2) were Cazenave and Weissler in 6]. There, they consider the space

$$
X_{\rho}=\left\{u \in L_{l o c}^{\infty}\left((0, \infty), L^{\rho+2}\left(\mathbb{R}^{n}\right)\right) \mid \sup _{t>0} t^{\frac{\alpha}{2}}\|u(t)\|_{L^{\rho+2}}<\infty\right\},
$$

where $\frac{\alpha}{2}=\frac{1}{\rho}-\frac{n}{2(\rho+2)}$ and $\|\cdot\|_{L^{\rho+2}}$ denotes the usual $L^{\rho+2}$ norm. Under a suitable smallness condition on the initial data, they prove the existence of global solutions of (1.1)-1.2) in $X_{\rho}$, for $\rho$ in the range

$$
\frac{\rho+2}{\rho+1}<\frac{n \rho}{2}<\rho+2 .
$$

If $n=1$ or $n=2$, condition (1.6) is equivalent to $\rho_{0}<\rho<\infty$, where $\rho_{0}$ is the positive value of $\rho$ for which $\frac{\rho+2}{\rho+1}=\frac{n \rho}{2}$. If $n \geq 3$, it is equivalent to $\rho_{0}<\rho<\frac{4}{n-2}$. Later on, in [2], the Cauchy problem (1.1)-(1.2) was studied in the framework of weak- $L^{p}$ spaces. Using a Strichartz-type inequality, the authors obtained existence of solutions in the class $L^{(p, \infty)}\left(\mathbb{R}^{n+1}\right) \equiv L_{t}^{(p, \infty)}\left(L_{x}^{(p, \infty)}\right)$, where $(t, x) \in \mathbb{R} \times \mathbb{R}^{n}$ and $p=\frac{\rho(n+2)}{2}$, for $\rho$ in the range

$$
\rho_{0}<\frac{4(n+1)}{n(n+2)}<\rho<\frac{4(n+1)}{n^{2}}<\frac{4}{n-2} .
$$

In [15], the existence of solutions with initial data in the Besov space $\dot{B}_{2}^{s_{\rho}, \infty}\left(\mathbb{R}^{n}\right)$, with positive regularity $s_{\rho}=\frac{n}{2}-\frac{2}{\rho}>0$, was proved for $\rho$ in the range $\rho_{0}<\frac{4}{n}<$ $\rho<\infty$. Note that if $f \in \dot{B}_{2}^{s_{\rho}, \infty}\left(\mathbb{R}^{n}\right)$, then $f$ has at least local finite energy.

We study equation (1.3) in functional spaces of infinite energy. In the first theorem proved here, we consider the initial data $\phi$ belonging to the Marcinkiewicz space $L^{\left(\frac{\rho+2}{\rho+1}, \infty\right)}$ and show existence and uniqueness of local in time solutions in the class

$$
E_{\alpha, \beta}^{T}=\left\{\left.u\left|\|u\|_{\alpha, \beta}=\sup _{-T<t<T}\right| t\right|^{\frac{\alpha-\beta}{2}}\|u(t)\|_{(\rho+2, \infty)}<\infty\right\}
$$

where $\frac{(\alpha-\beta)}{2}=\frac{n \rho}{2(\rho+2)}$, with $\frac{n \rho}{2}<\frac{\rho+2}{\rho+1}$. Note that $\frac{n \rho}{2}<\frac{\rho+2}{\rho+1}$ is equivalent to $0<\rho<\rho_{0}<\frac{4}{n}$. So, our range for $\rho$ is different from the ones in [6, 2, 15]. The norm $\|\cdot\|_{\alpha, \beta}$ is not invariant by the scaling $u_{\mu}(t, x)=\mu^{\frac{2}{\rho}} u\left(\mu^{2} t, \mu x\right)$. This is a key point to obtain local in time solutions in Marcinkiewicz spaces. It is also worth noting that our result allows one to consider singular initial data as, for example, homogeneous functions $|x|^{-\frac{n(\rho+1)}{\rho+2}} \in L^{\left(\frac{\rho+2}{\rho+1}, \infty\right)}$.

Our second theorem concerns global in time solutions. We show existence of such solutions in norms of type $\sup _{|t|>0}|t|^{\alpha / 2}\|u(t)\|_{L^{(\rho+2, \infty)}}$, where $\frac{\alpha}{2}=\frac{1}{\rho}-\frac{n}{2(\rho+2)}$ and

$$
\rho_{0}<\rho<\frac{4}{n-2}
$$

This extends the result of Cazenave and Weissler [6] to the context of Lorentz spaces. Note that range (1.8) is greater than range (1.7).

As a corollary, we show that when the initial data $\phi$ is a homogeneous function of degree $-\frac{2}{\rho}$, we obtain a self-similar solution if $\|S(1) \phi\|_{(\rho+2, \infty)}$ is sufficiently small. Moreover, we discuss asymptotic stability of the global solutions and show that regular perturbations of the linear Schrödinger equations are negligible for large times. We also analyze the behavior of the local solutions as $t \rightarrow 0$ in $L^{(\rho+2, \infty)}$. 
Our approach is different from the methods used in 2, 15, where the authors use a Strichartz-type inequality in weak- $L^{p}$ and Besov spaces, respectively. Indeed, our existence results are based on bounds for the Schrödinger linear group $S(t)$ in the context of Lorentz spaces. In Lemma 2.1, we state and prove these bounds via real interpolation techniques. They generalize the bounds for the usual $L^{p}$ spaces used in [6].

In section 2, we carefully state our results and discuss their improvement in the light of previous results. We prove them in section 3

\section{MAIn RESUlts}

We first recall some facts about interpolation in Lorentz spaces. For more properties and details about these spaces see, for instance, 1 and [16. The Lorentz spaces $L^{(p, q)}\left(\mathbb{R}^{n}\right)$ can be constructed via real interpolation. Indeed, $L^{(p, q)}\left(\mathbb{R}^{n}\right)=$ $\left(L^{1}\left(\mathbb{R}^{n}\right), L^{\infty}\left(\mathbb{R}^{n}\right)\right)_{1-\frac{1}{p}, q}, 1<p<\infty$. They have the interpolation property

$$
\left(L^{\left(p_{0}, q_{0}\right)}\left(\mathbb{R}^{n}\right), L^{\left(p_{1}, q_{1}\right)}\left(\mathbb{R}^{n}\right)\right)_{\theta, q}=L^{(p, q)}\left(\mathbb{R}^{n}\right),
$$

provided $0<p_{0}<p_{1}<\infty, 0<\theta<1, \frac{1}{p}=\frac{1-\theta}{p_{0}}+\frac{\theta}{p_{1}}, 1 \leq q_{0}, q_{1}, q \leq \infty$, where $(\cdot, \cdot)_{\theta, q}$ stands for the real interpolation spaces constructed via the $K$-method [1]. The spaces $L^{(p, \infty)}$ are called either weak- $L^{p}$ or Marcinkiewicz spaces.

We begin by bounding the Schrödinger group $S(t)$ in Lorentz spaces.

Lemma 2.1. Let $1 \leq d \leq \infty$, and $1<p<2$. If $p^{\prime}$ is such that $\frac{1}{p}+\frac{1}{p^{\prime}}=1$, then there exists a constant $C=C(n, p)>0$ such that

$$
\|S(t) \varphi\|_{\left(p^{\prime}, d\right)} \leq C|t|^{-\frac{n}{2}\left(\frac{2}{p}-1\right)}\|\varphi\|_{(p, d)},
$$

for all $\varphi \in L^{(p, d)}\left(\mathbb{R}^{n}\right)$ and all $t \neq 0$.

Proof. Fix $t \neq 0$ and let $1<p_{0}<p<p_{1}<2$ such that $\frac{1}{p^{\prime}}=\frac{\lambda}{p_{0}}+\frac{1-\lambda}{p_{1}}$ and $0<\lambda<1$. By the well-known $L^{p}=L^{(p, p)}$ estimate of the Schrödinger group, we have $S(t): L^{p_{0}} \rightarrow L^{p_{0}^{\prime}}$ and $S(t): L^{p_{1}} \rightarrow L^{p_{1}^{\prime}}$, with operator norms bounded by

$$
\|S(t)\|_{p_{0} \rightarrow p_{0}^{\prime}} \leq C|t|^{-\frac{n}{2}\left(\frac{2}{p_{0}}-1\right)},\|S(t)\|_{p_{1} \rightarrow p_{1}^{\prime}} \leq C|t|^{-\frac{n}{2}\left(\frac{2}{p_{1}}-1\right)} .
$$

Through real interpolation,

$$
\begin{aligned}
\|S(t)\|_{(p, d) \rightarrow\left(p^{\prime}, d\right)} & \leq\|S(t)\|_{p_{0} \rightarrow p_{0}^{\prime}}^{\lambda}\|S(t)\|_{p_{1} \rightarrow p_{1}^{\prime}}^{1-\lambda} \\
& \leq\left(C|t|^{-\frac{n}{2}\left(\frac{2}{p_{0}}-1\right)}\right)^{\lambda}\left(C|t|^{-\frac{n}{2}\left(\frac{2}{p_{1}}-1\right)}\right)^{1-\lambda}=C|t|^{-\frac{n}{2}\left(\frac{2}{p}-1\right)},
\end{aligned}
$$

which is equivalent to (2.1).

From now on, we denote $\alpha:=\frac{2}{\rho}-\frac{n}{\rho+2}$ and $\beta:=\frac{2}{\rho}-\frac{n(\rho+1)}{(\rho+2)}$.

Definition 2.2. Let $0<\rho<\infty$ and $0<T \leq \infty$. We denote by $E_{\alpha}$ and $E_{\alpha, \beta}^{T}$ the Banach spaces

$$
\begin{aligned}
E_{\alpha} & =\left\{\left.u|| t\right|^{\frac{\alpha}{2}} u \in B C\left((-\infty, \infty) ; L^{(\rho+2, \infty)}\right)\right\}, \\
E_{\alpha, \beta}^{T} & =\left\{\left.u|| t\right|^{\frac{\alpha-\beta}{2}} u \in B C\left((-T, T) ; L^{(\rho+2, \infty)}\right)\right\},
\end{aligned}
$$


with respective norms

$$
\|u\|_{\alpha}=\sup _{-\infty<t<\infty}|t|^{\frac{\alpha}{2}}\|u(t)\|_{(\rho+2, \infty)},\|u\|_{\alpha, \beta}=\sup _{-T<t<T}|t|^{\frac{\alpha-\beta}{2}}\|u(t)\|_{(\rho+2, \infty)},
$$

which are weakly continuous in the sense of distributions at $t=0$.

Definition 2.3. Let $0<T \leq \infty$. A mild solution of the initial value problem (1.1)(1.2) in the space $E_{\alpha, \beta}^{T}$ (respectively, in the space $E_{\alpha}$ ) is a complex-valued function $u \in E_{\alpha, \beta}^{T}$ (respectively, $u \in E_{\alpha}$ ) satisfying equation (1.3) for all $0<|t|<T$, such that $u(t) \rightarrow \phi$ when $t \rightarrow 0$ in the sense of distributions.

Our main results are

Theorem 2.4 (Local in time solutions). Let $0<\rho<\infty$ and $\frac{n \rho}{2}<\frac{\rho+2}{\rho+1}$.

(1) If $\phi \in L^{\left(\frac{\rho+2}{\rho+1}, \infty\right)}$, then there exists $0<T<\infty$ such that the initial value problem (1.1)-(1.2) has a unique mild solution $u(t, x) \in E_{\alpha, \beta}^{T}$, with $T=$ $T(\phi)=C\|\phi\|_{\left(\frac{\rho+2}{\rho+1}, \infty\right)}^{-\frac{\rho}{\delta}}$, where $\delta=1-\frac{\alpha-\beta}{2}(\rho+1)>0$.

(2) Moreover, if $\phi_{n} \in L^{\left(\frac{\rho+2}{\rho+1}, \infty\right)}$ is a sequence of functions satisfying $\phi_{n} \rightarrow \phi$ in $L^{\left(\frac{\rho+2}{\rho+1}, \infty\right)}$, then there exists $0<T_{0}<\infty$ and $n_{0} \in \mathbb{N}$ such that, for $n \geq n_{0}$, the solutions $u_{n}$ and $u$ with respective initial data $\phi_{n}$ and $\phi$ lie in $E_{\alpha, \beta}^{T_{0}}$ and $u_{n} \rightarrow u$ in $E_{\alpha, \beta}^{T_{0}}$. Actually, the solution map $\phi \mapsto u$ is Lipschitz continuous.

Theorem 2.5 (Global in time solutions). Let $0<\rho<\infty$ and $\frac{\rho+2}{\rho+1}<\frac{n \rho}{2}<\rho+2$.

(1) If $\phi$ is a distribution such that $\sup _{-\infty<t<\infty}|t|^{\frac{\alpha}{2}}\|S(t) \phi\|_{(\rho+2, \infty)}<\varepsilon$, for $\varepsilon>0$ small enough, then problem (1.1)-(1.2) has a global in time mild solution $u(t, x) \in E_{\alpha}$. This solution is the only one satisfying $\|u\|_{\alpha} \leq 2 \varepsilon$.

(2) Furthermore, if $\left(\phi_{n}\right)$ is a sequence of distributions such that $\| S(t) \phi_{n}$ $S(t) \phi \|_{E_{\alpha}} \rightarrow 0$ when $n \rightarrow \infty$, and $u_{n}, u$ are the solutions with respective initial data $\phi_{n}$ and $\phi$, then $u_{n} \rightarrow u$ in $E_{\alpha}$.

We compare the theorems above with previous results.

- In [6], the existence of solutions in spaces of infinite energy was obtained for $\rho_{0}<\rho<\frac{4}{n-2}$, where $\rho_{0}$ is the value of $\rho$ for which $\frac{\rho+2}{\rho+1}=\frac{n \rho}{2}$. In [2, using Strichartz-type inequalities, the existence of global solutions in the class $L^{(p, \infty)}\left(\mathbb{R}^{n+1}\right) \equiv L_{t}^{(p, \infty)}\left(L_{x}^{(p, \infty)}\right)$ was established, where $p=\frac{\rho(n+2)}{2}$ and $\rho_{0}<\frac{4(n+1)}{n(n+2)}<\rho<\frac{4(n+1)}{n^{2}}$. So, Theorem 2.4 extends the set of exponents $\rho$ where such solutions exist by including the interval $0<\rho<\rho_{0}$.

- In the range $\rho_{0}<\rho<\frac{4}{n-2}$, Theorem 2.5 extends the global solutions results derived in [6] to the framework of Marcinkiewicz spaces. Our range for $\rho$ is also greater than the one in [2] (see (1.7)).

- Theorem 2.4 assures the existence of local in time solutions even for singular initial data $\phi(x)=P_{k}(x)|x|^{-k-\frac{n(\rho+1)}{\rho+2}} \in L^{\left(\frac{\rho+2}{\rho+1}, \infty\right)}$, where $P_{k}(x)$ is a homogeneous polynomial of degree $k$. As far as we know, there were no previous existence results covering this case. On the other hand, we were not able to obtain self-similar solutions in $E_{\alpha, \beta}$ though, since the norm $\|\cdot\|_{\alpha, \beta}$ is not invariant by the scaling relation $u_{\mu}(t, x)=\mu^{\frac{2}{\rho}} u\left(\mu^{2} t, \mu x\right)$. 
- In [7, 14, 17, there are results for Schrödinger type equations in various energy spaces, different than the ones considered here, through diverse approaches. In particular, in [7] local weak solutions for problem (1.1)-(1.2), $n=1, \rho=2$, were studied in Sobolev spaces $H^{s}$, for $s$ negative but close to 0 . In [14, the authors have studied a Schrödinger equation with a nonlinear term involving derivatives, assuming periodic initial data satisfying $\left\|\left(1+\xi^{2}\right)^{s / 2} \hat{\phi}\right\|_{l_{\xi}^{p}}<\infty$ with positive regularity $s \geq 1 / 2$ and $p \in(2,4)$. In [17, the authors have gotten solutions for problem (1.1)-(1.2), with $n=1$, $\rho=2$, and initial data satisfying $\|S(t) \phi\|_{L^{3}\left((-T, T) ; L^{6}\right)}<\infty$. Results of the type $L^{2}-H^{s}$ are related to those derived in [6, 2, 15, for more general spaces, such as Besov, $L^{p}$, and weak- $L^{p}$. In our case, when $\rho_{0}<\rho<\frac{4}{n-2}$, if one takes initial data $\phi \in H^{1}$, then, since

$$
\|S(t) \phi\|_{(\rho+2, \infty)} \leq C\|S(t) \phi\|_{H^{1}}=C\|\phi\|_{H^{1}},
$$

there exists $T_{0}>0$ such that the unique global small solution belongs to the class $C\left(\left[-T_{0}, T_{0}\right] ; H^{1}\left(\mathbb{R}^{n}\right)\right)$.

As a direct consequence of Theorem 2.5, one can show the existence of a self-similar solution.

Corollary 2.6 (Self-similar solutions). In addition to the hypothesis of Theorem 2.5, if the initial data $\phi$ is a sufficiently small homogeneous function of degree $-\frac{2}{\rho}$, then the solution $u(t, x)$ provided by Theorem 2.5 is self-similar, that is, $u(t, x)=$ $\mu^{\frac{2}{\rho}} u\left(\mu^{2} t, \mu x\right)$ for all $\mu>0$, almost everywhere for $x \in \mathbb{R}^{n}$ and $t>0$.

Remark 2.7. Let $P_{k}(x)$ be a homogeneous polynomial of degree $k$. The set of functions $\phi$ which are finite linear combinations of functions of the form $\frac{P_{k}(x)}{|x|^{k+\frac{2}{\rho}}}$ is an admissible class for the existence of self-similar solutions for problem (1.1)-(1.2).

We also analyze the large time behaviour of the solutions given by Theorem 2.5 and study the behaviour of the solutions given in Theorem 2.4 near to time $t=0$. These are the content of the following theorem.

Theorem 2.8. (1) (Asymptotic stability) Suppose $0 \leq h<1-\frac{\alpha}{2}(\rho+1)$, and let $u, v \in E_{\alpha}$ be two global solutions of problem (1.1)-(1.2) obtained through Theorem 2.5, corresponding to respective initial conditions $\phi, \varphi$. If $\lim _{|t| \rightarrow \infty}|t|^{\frac{\alpha}{2}+h}\|S(t)(\phi-\varphi)\|_{(\rho+2, \infty)}=0$, then

$$
\lim _{|t| \rightarrow \infty}|t|^{\frac{\alpha}{2}+h}\|u(t)-v(t)\|_{(\rho+2, \infty)}=0 .
$$

(2) (Decay rate as $t \rightarrow 0$ ) Suppose $\delta=1-\frac{\alpha-\beta}{2}(\rho+1)>0$, and $h>-\delta$. Let $u, v \in E_{\alpha, \beta}$ be two local solutions of (1.1)-(1.2) obtained through Theorem 2.4, corresponding to initial conditions $\phi, \varphi \in L^{\left(\frac{\rho+2}{\rho+1}, \infty\right)}$, respectively.

$$
\text { If } \begin{aligned}
& \lim _{t \rightarrow 0}|t|^{\frac{\alpha-\beta}{2}-h} \\
& \qquad S(t)(\phi-\varphi) \|_{(\rho+2, \infty)}=0 \text {, then } \\
& \qquad \lim _{t \rightarrow 0}|t|^{\frac{\alpha-\beta}{2}-h}\|u(t)-v(t)\|_{(\rho+2, \infty)}=0 .
\end{aligned}
$$

Let us comment on some improvements produced by Theorem 2.8 , 
- (Asymptotic stability) Theorem 2.5 already gives

$$
\sup _{|t|>0}|t|^{\frac{\alpha}{2}}\|u(t)-v(t)\|_{(\rho+2, \infty)}<\infty \text {. }
$$

Thus, it is obvious that estimate (2.4) holds for $h<0$. On the other hand, the first item in Theorem 2.8 extends this property for the range $0 \leq h<1-\frac{\alpha}{2}(\rho+1)$. However, more regularity on the initial perturbation $\phi-\varphi$ is required. For instance, assuming (in addition) that $\phi-\varphi \in L^{\left(\frac{\rho+2}{\rho+1}, \infty\right)}$, one obtains

$\lim _{|t| \rightarrow \infty}|t|^{h+\frac{\alpha}{2}}\|S(t)(\phi-\varphi)\|_{(\rho+2, \infty)}=\|\phi-\varphi\|_{\left(\frac{\rho+2}{\rho+1}, \infty\right)} \lim _{|t| \rightarrow \infty}|t|^{h+\beta / 2}=0$,

with $0 \leq h<-\frac{\beta}{2}$. Observe that $-\frac{\beta}{2}=1-\frac{\alpha}{2}(\rho+1)>0$, when $\rho_{0}<\rho<\frac{4}{n-2}$.

- (Decay rate when $t \rightarrow 0$ ) By bound (3.2), one can see that

$|t|^{\frac{\alpha-\beta}{2}-h}\|u(t)-v(t)\|_{(\rho+2, \infty)} \leq|t|^{\frac{\alpha-\beta}{2}-h}\|S(t)(\phi-\varphi)\|_{(\rho+2, \infty)}+C|t|^{\delta-h}$,

which implies the bound (2.5) for $h<\delta$. Assuming further regularity for $\phi-\varphi$, the second item of Theorem 2.8 extends this property for the range $h>-\delta$.

- In Theorem 2.8, if one assumes the stronger hypothesis

$$
\lim _{|t| \rightarrow \infty}|t|^{\frac{\alpha}{2}+h}\|S(t)(\phi-\varphi)\|_{L^{\rho+2}}=0
$$

then using the existence theorem of [6] and arguing as in the proof of the first part of Theorem 2.8, one can show that

$$
\lim _{|t| \rightarrow \infty}|t|^{\frac{\alpha}{2}+h}\|u(t)-v(t)\|_{L^{\rho+2}}=0 .
$$

- In [6], assuming $\phi-\varphi \in L^{\frac{\rho+2}{\rho+1}}\left(\mathbb{R}^{n}\right)$ and $0 \leq h<1-\frac{\alpha}{2}(\rho+1)$, the authors obtained that $\|u(t)-v(t)\|_{L^{\rho+2}}=\mathcal{O}\left(t^{-\left(\frac{\alpha}{2}+h\right)}\right)$. Inequality (2.6) is sharper, i.e., $\|u(t)-v(t)\|_{L^{\rho+2}}=o\left(t^{-\left(\frac{\alpha}{2}+h\right)}\right)$.

\section{Proofs}

The following lemma is important to our ends. For its proof, see [8].

Lemma 3.1. Let $0<\rho<\infty$ and let $X$ be a Banach space with norm $\|\cdot\|$. Suppose $B: X \rightarrow X$ to be a map satisfying

$$
\|B(x)-B(z)\| \leq K\|x-z\|\left(\|x\|^{\rho}+\|z\|^{\rho}\right),
$$

$B(0)=0$, and let $R>0$ be the unique positive root of equation $2^{\rho+1} K(R)^{\rho}-1=0$. Given $0<\varepsilon<R$ and $y \in X, y \neq 0$, such that $\|y\| \leq \varepsilon$, there exists a solution $x \in X$ for the equation $x=y+B(x)$ such that $\|x\| \leq 2 \varepsilon$. The solution $x$ is unique in the ball $B_{2 \varepsilon}:=\bar{B}(0,2 \varepsilon)$. Moreover, the solution depends continuously on $y$ in the following sense: If $\|\tilde{y}\| \leq \varepsilon, \tilde{x}=\tilde{y}+B(\tilde{x})$, and $\|\tilde{x}\| \leq 2 \varepsilon$, then

$$
\|x-\tilde{x}\| \leq \frac{1}{1-2^{\rho+1} K \varepsilon^{\rho}}\|y-\tilde{y}\| .
$$

Now, we state and prove the necessary estimates in order to apply Lemma 3.1 in our case. 
Lemma 3.2. Let $0<\rho<\infty$ and $B$ be defined as

$$
B(u)=-i \lambda \int_{0}^{t} S(t-s)\left(|u(s)|^{\rho} u(s)\right) d s .
$$

If $\frac{n \rho}{2}<\frac{\rho+2}{\rho+1}$, then there exists a positive constant $K_{\alpha, \beta}$ such that

$$
\|B(u)-B(v)\|_{\alpha, \beta} \leq K_{\alpha, \beta} T^{1-\frac{(\alpha-\beta)(\rho+1)}{2}}\|u-v\|_{\alpha, \beta}\left(\|u\|_{\alpha, \beta}^{\rho}+\|v\|_{\alpha, \beta}^{\rho}\right),
$$

for all $u, v \in E_{\alpha, \beta}^{T}$. On the other hand, if $\frac{\rho+2}{\rho+1}<\frac{n \rho}{2}<\rho+2$, then there exists a positive constant $K_{\alpha}$ such that

$$
\|B(u)-B(v)\|_{\alpha} \leq K_{\alpha}\|u-v\|_{\alpha}\left(\|u\|_{\alpha}^{\rho}+\|v\|_{\alpha}^{\rho}\right),
$$

for all $u, v \in E_{\alpha}$.

Proof. Without loss of generality, we assume $t>0$. First note that if $\frac{n \rho}{2}<\frac{\rho+2}{\rho+1}<$ $\rho+2$, then $\frac{\alpha-\beta}{2}(\rho+1)<1$ and $\frac{n}{2}\left(\frac{2(\rho+1)}{\rho+2}-1\right)<1$. Therefore,

$$
\begin{aligned}
& \|B(u)-B(v)\|_{(\rho+2, \infty)} \leq \int_{0}^{t}\left\|S(t-s)\left(|u|^{\rho} u-|v|^{\rho} v\right)\right\|_{(\rho+2, \infty)} d s \\
& \leq C \int_{0}^{t}(t-s)^{-\frac{n}{2}\left(\frac{2(\rho+1)}{\rho+2}-1\right)}\left\|(|u-v|)\left(|u|^{\rho}+|v|^{\rho}\right)\right\|_{\left(\frac{\rho+2}{\rho+1}, \infty\right)} d s \\
& \leq C \int_{0}^{t}(t-s)^{-\frac{n}{2}\left(\frac{2(\rho+1)}{\rho+2}-1\right)}\|u-v\|_{(\rho+2, \infty)}\left(\|u\|_{(\rho+2, \infty)}^{\rho}+\|v\|_{(\rho+2, \infty)}^{\rho}\right) d s \\
& \left.\leq C \sup _{0<t<T} t^{\frac{\alpha-\beta}{2}}\|u-v\|_{(\rho+2, \infty)} \sup _{0<t<T}\left(t^{\frac{(\alpha-\beta) \rho}{2}}\|u\|_{(\rho+2, \infty)}^{\rho}+t^{\frac{(\alpha-\beta) \rho}{2}}\|v\|_{(\rho+2, \infty)}^{\rho}\right)\right) \\
& \quad \cdot \int_{0}^{t}(t-s)^{-\frac{\alpha-\beta}{2}} s^{-\frac{\alpha-\beta}{2}(\rho+1)} d s \\
& =K_{\alpha, \beta} t^{-\frac{\alpha-\beta}{2}} t^{1-\frac{\alpha-\beta}{2}(\rho+1)}\|u-v\|_{\alpha, \beta}\left(\|u\|_{\alpha, \beta}^{\rho}+\|v\|_{\alpha, \beta}^{\rho}\right),
\end{aligned}
$$

which proves (3.2). On the other hand, if $\frac{\rho+2}{\rho+1}<\frac{n \rho}{2}<\rho+2$, then $\frac{\alpha}{2}(\rho+1)<1$ and $\frac{n}{2}\left(\frac{2(\rho+1)}{\rho+2}-1\right)<1$. In this case,

$$
\begin{aligned}
\| B(u) & -B(v) \|_{(\rho+2, \infty)} \\
& \leq C \int_{0}^{t}(t-s)^{-\frac{n}{2}\left(\frac{2(\rho+1)}{\rho+2}-1\right)}\|u-v\|_{(\rho+2, \infty)}\left(\|u\|_{(\rho+2, \infty)}^{\rho}+\|v\|_{(\rho+2, \infty)}^{\rho}\right) d s \\
& \leq C\left(\sup _{t>0} t^{\frac{\alpha}{2}}\|u-v\|_{(\rho+2, \infty)} \sup _{t>0}\left(t^{\frac{\alpha \rho}{2}}\|u\|_{(\rho+2, \infty)}^{\rho}+t^{\frac{\alpha \rho}{2}}\|v\|_{(\rho+2, \infty)}^{\rho}\right)\right) \\
& \quad \cdot \int_{0}^{t}(t-s)^{-\frac{n}{2}\left(\frac{2(\rho+1)}{\rho+2}-1\right)} s^{-\frac{\alpha}{2}(\rho+1)} d s \\
&
\end{aligned}
$$

which proves (3.3). 
3.1. Proof of Theorem 2.4, Let $y=S(t) \phi$. Due to Lemma 2.1, one has

$$
\|y\|_{\alpha, \beta}=\sup _{-T<t<T}|t|^{\frac{\alpha-\beta}{2}}\|S(t) \phi\|_{(\rho+2, \infty)} \leq C\|\phi\|_{\left(\frac{\rho+2}{\rho+1}, \infty\right)}<\infty .
$$

Using Lemma 3.2, one gets

$$
\|B(u)-B(v)\|_{\alpha, \beta} \leq K_{\alpha, \beta} T^{\delta}\|u-v\|_{\alpha, \beta}\left(\|u\|_{\alpha, \beta}^{\rho}+\|v\|_{\alpha, \beta}^{\rho}\right),
$$

where $\delta=1-\frac{\alpha-\beta}{2}(\rho+1)>0$. Now, choose $0<T<\infty$ sufficiently small, and $\varepsilon>0$ such that $\|y\|_{\alpha, \beta} \leq C\|\phi\|_{\left(\frac{\rho+2}{\rho+1}, \infty\right)}=\varepsilon<R:=\left(\frac{1}{2^{(\rho+1)} K_{\alpha, \beta} T^{\delta}}\right)^{\frac{1}{\rho}}$. Using Lemma 3.1 with $X=E_{\alpha, \beta}^{T}$, one assures the existence of a local mild solution $u \in E_{\alpha, \beta}^{T}$. Moreover, this solution is unique in the ball $B_{2 \varepsilon}:=\bar{B}(0,2 \varepsilon) \subset E_{\alpha, \beta}^{T}$. Furthermore, through standard arguments, one can prove that $u(t) \rightarrow \phi$ in the sense of distributions when $t \rightarrow 0$. So, solutions of the integral equation are indeed mild solutions in the sense of Definition 2.3.

Finally, let $u_{n}$ and $u$ be the solutions with respective initial data $\phi_{n}$ and $\phi$. Lemma 3.1 gives

$$
\begin{aligned}
\left\|u_{n}-u\right\|_{E_{\alpha, \beta}} & \leq \frac{1}{1-2^{\rho+1} K_{\alpha, \beta} T^{\delta} \varepsilon^{\rho}}\left\|S(t) \phi_{n}-S(t) \phi\right\|_{E_{\alpha, \beta}} \\
& \leq \frac{C}{1-2^{\rho+1} K_{\alpha, \beta} T^{\delta} \varepsilon^{\rho}}\left\|\phi_{n}-\phi\right\|_{\left(\frac{\rho+2}{\rho+1}, \infty\right)} .
\end{aligned}
$$

This finishes the proof.

3.2. Proof of Theorem 2.5. Apply Lemma 3.1 to the integral equation (1.3) with $X=E_{\alpha}$ and $y=S(t) \phi$. In this case, bound (3.3) gives

$$
\|B(u)-B(v)\|_{\alpha} \leq K_{\alpha}\|u-v\|_{\alpha}\left(\|u\|_{\alpha}^{\rho}+\|v\|_{\alpha}^{\rho}\right) .
$$

Now, one considers $\varepsilon>0$ small enough so that

$$
\|S(t) \phi\|_{\alpha}=\sup _{|t|>0}|t|^{\frac{\alpha}{2}}\|S(t) \phi\|_{(\rho+2, \infty)}<\varepsilon
$$

allows one to apply Lemma 3.1 repeatedly, in order to obtain the existence of a global mild solution $u \in E_{\alpha}$. This solution is unique in the ball $B_{2 \varepsilon}:=\bar{B}(0,2 \varepsilon) \subset$ $E_{\alpha}$.

The continuity of the solutions with respect to the initial conditions, as well as the continuity of the solutions in the sense of distributions, follows as in the proof of Theorem 2.4.

3.3. Proof of Corollary 2.6. Let $t>0$. If the initial data $\phi(x)$ is a homogeneous function of degree $-\frac{2}{\rho}$, then $S(t) \phi$ satisfies the self-similar property $u(t, x)=$ $\mu^{\frac{2}{\rho}} u\left(\mu^{2} t, \mu x\right)$. Thus,

$$
t^{\alpha / 2}\|S(t) \phi\|_{(\rho+2, \infty)}=t^{\frac{\alpha}{2}} t^{\frac{n}{2(\rho+2)}-\frac{1}{\rho}}\|S(1) \phi\|_{(\rho+2, \infty)}=\|S(1) \phi\|_{(\rho+2, \infty)} .
$$

Moreover, $\|S(1) \phi\|_{L^{\rho+2}}$ is finite (see [6]). Since $L^{\rho+2} \hookrightarrow L^{(\rho+2, \infty)}$ continuously, one has $\|S(1) \phi\|_{(\rho+2, \infty)} \leq\|S(1) \phi\|_{L^{\rho+2}}<\infty$. Therefore, if $\|S(1) \phi\|_{(\rho+2, \infty)}$ is small enough, it is straightforward to show that the solution $u(t, x)$ obtained in Theorem 2.4 is self-similar. 
3.4. Proof of Theorem 2.8. Without loss of generality, assume $t>0$. Taking the difference of the integral equations satisfied by $u$ and $v$, one gets

$$
\begin{aligned}
t^{\frac{\alpha}{2}+h}\|u(t)-v(t)\|_{(\rho+2, \infty)} & \leq t^{\frac{\alpha}{2}+h}\|S(t)(\phi-\varphi)\|_{(\rho+2, \infty)} \\
& +t^{\frac{\alpha}{2}+h}\left\|\int_{0}^{t} S(t-s)\left(u|u|^{\rho}-v|v|^{\rho}\right) d s\right\|_{(\rho+2, \infty)} .
\end{aligned}
$$

Since $\|u\|_{\alpha},\|v\|_{\alpha} \leq 2 \varepsilon$, one uses the change of variable $s \longmapsto t s$ and bounds

$$
\begin{aligned}
& t^{\frac{\alpha}{2}+h}\left\|\int_{0}^{t} S(t-s)\left(u|u|^{\rho}-v|v|^{\rho}\right) d s\right\|_{(\rho+2, \infty)} \\
& \leq C t^{\frac{\alpha}{2}+h} \int_{0}^{t}\left[(t-s)^{-\frac{n}{2}\left(\frac{2(\rho+1)}{(\rho+2)}-1\right)} s^{-\frac{\alpha(\rho+1)}{2}-h}\right. \\
& \left.\quad \cdot\left(s^{\frac{\alpha \rho}{2}}\|u(s)\|_{(\rho+2, \infty)}^{\rho}+s^{\frac{\alpha \rho}{2}}\|v(s)\|_{(\rho+2, \infty)}^{\rho}\right)\left(s^{\frac{\alpha}{2}+h}\|u(s)-v(s)\|_{(\rho+2, \infty)}\right)\right] d s \\
& \leq C 2^{\rho+1} \varepsilon^{\rho} \int_{0}^{1}(1-s)^{-\frac{n}{2}\left(\frac{2(\rho+1)}{(\rho+2)}-1\right)} s^{-\frac{\alpha(\rho+1)}{2}-h}(t s)^{\frac{\alpha}{2}+h}\|u(t s)-v(t s)\|_{(\rho+2, \infty)} d s .
\end{aligned}
$$

Therefore,

$$
\begin{aligned}
& t^{\frac{\alpha}{2}+h}\|u(t)-v(t)\|_{(\rho+2, \infty)} \leq t^{\frac{\alpha}{2}+h}\|S(t)(\phi-\varphi)\|_{(\rho+2, \infty)} \\
& \quad+C 2^{\rho+1} \varepsilon^{\rho} \int_{0}^{1}(1-s)^{-\frac{n}{2}\left(\frac{2(\rho+1)}{(\rho+2)}-1\right)} s^{-\frac{\alpha}{2}(\rho+1)-h}(t s)^{\frac{\alpha}{2}+h}\|u(t s)-v(t s)\|_{(\rho+2, \infty)} d s,
\end{aligned}
$$

for all $t>0$. Now, define

$$
A:=\limsup _{t \rightarrow \infty} t^{\frac{\alpha}{2}+h}\|u(t)-v(t)\|_{(\rho+2, \infty)} .
$$

Using the assumption on the initial perturbation $\phi-\varphi$, it is not difficult to show that $A<\infty$. Now, note that

$$
\begin{aligned}
& \limsup _{t \rightarrow \infty} \int_{0}^{1}(1-s)^{-\frac{n}{2}\left(\frac{2(\rho+1)}{(\rho+2)}-1\right)} s^{\frac{-\alpha(\rho+1)}{2}-h}(t s)^{\frac{\alpha}{2}+h}\|u(t s)-v(t s)\|_{(\rho+2, \infty)} d s \\
& \leq A \int_{0}^{1}(1-s)^{-\frac{n}{2}\left(\frac{2(\rho+1)}{(\rho+2)}-1\right)} s^{-\frac{\alpha}{2}(\rho+1)-h} d s .
\end{aligned}
$$

So, taking the $\limsup _{t \rightarrow \infty}$ in (3.5), one obtains

$$
A \leq\left(C 2^{\rho+1} \varepsilon^{\rho} \int_{0}^{1}(1-s)^{-\frac{n}{2}\left(\frac{2(\rho+1)}{(\rho+2)}-1\right)} s^{-\frac{\alpha(\rho+1)}{2}-h} d s\right) A .
$$

Now, let $\Gamma:=C 2^{\rho+1} \int_{0}^{1}(1-s)^{-\frac{n}{2}\left(\frac{2(\rho+1)}{(\rho+2)}-1\right)} s^{-\frac{\alpha(\rho+1)}{2}-h} d s$. Choosing $\varepsilon>0$ small enough such that $\varepsilon^{\rho} \Gamma<1$, one concludes that $A=0$. This proves part 1 of the theorem.

In order to prove part 2, let $\delta=1-\frac{\alpha-\beta}{2}(\rho+1)$ and $0<t<T$ as in Theorem 2.4, One can write $\delta=\frac{\alpha-\beta}{2}-h-\frac{\alpha-\beta}{2}-\frac{\alpha-\beta}{2}(\rho+1)+h+1$. Again, one subtracts the 
equations for $u$ and $v$ and bounds

$$
\begin{gathered}
t^{\frac{\alpha-\beta}{2}-h}\left\|\int_{0}^{t} S(t-s)\left(u|u|^{\rho}-v|v|^{\rho}\right) d s\right\|_{(\rho+2, \infty)} \\
\leq C t^{\frac{\alpha-\beta}{2}}-h \int_{0}^{t}\left[(t-s)^{-\frac{\alpha-\beta}{2}} s^{-\frac{\alpha-\beta}{2}(\rho+1)+h}\left(s^{\frac{\alpha-\beta}{2} \rho}\left(\|u(s)\|_{(\rho+2, \infty)}^{\rho}+\|v(s)\|_{(\rho+2, \infty)}^{\rho}\right)\right)\right. \\
\left.\leq C 2^{\rho+1} \varepsilon^{\rho} t^{\frac{\alpha-\beta}{2}-h-\frac{\alpha-\beta}{2}-\frac{\alpha-\beta}{2}(\rho+1)+h+1} \cdot s^{\frac{\alpha-\beta}{2}-h}\|u(s)-v(s)\|_{(\rho+2, \infty)}\right] d s \\
\cdot \int_{0}^{1}(1-s)^{-\frac{\alpha-\beta}{2}} s^{-\frac{\alpha-\beta}{2}(\rho+1)+h}(t s)^{\frac{\alpha-\beta}{2}-h}\|u(t s)-v(t s)\|_{(\rho+2, \infty)} d s .
\end{gathered}
$$

Hence,

$$
\begin{aligned}
t^{\frac{\alpha-\beta}{2}}-h & \|u(t)-v(t)\|_{(\rho+2, \infty)} \leq t^{\frac{\alpha-\beta}{2}-h}\|S(t)(\phi-\varphi)\|_{(\rho+2, \infty)} \\
& +C 2^{\rho+1} \varepsilon^{\rho} t^{\delta} \int_{0}^{1}(1-s)^{-\frac{\alpha-\beta}{2}} s^{-\frac{\alpha-\beta}{2}(\rho+1)+h}(t s)^{\frac{\alpha-\beta}{2}-h}\|u(t s)-v(t s)\|_{(\rho+2, \infty)} d s .
\end{aligned}
$$

Writing $A:=\lim \sup _{t \rightarrow 0} t^{\frac{\alpha-\beta}{2}-h}\|u(t)-v(t)\|_{(\rho+2, \infty)}<\infty$, one takes the lim $\sup _{t \rightarrow 0}$ in the last inequality to obtain

$$
0 \leq A \leq C 2^{\rho+1} \varepsilon^{\rho} A \int_{0}^{1}(1-s)^{-\frac{\alpha-\beta}{2}} s^{-\frac{\alpha-\beta}{2}(\rho+1)+h} d s \lim _{t \rightarrow 0} t^{\delta}=0 .
$$

This concludes the proof.

\section{REFERENCES}

[1] Bergh, J., Löfström, J., Interpolation Spaces. An Introduction, Springer, Grundlehren der Mathematischen Wissenschaften, No. 223, Springer-Verlag, Berlin-New York, 1976. MR 0482275 (58:2349)

[2] Cazenave, T., Vega, L., Vilela, M. C., A note on the nonlinear Schrödinger equation in weak $L^{p}$ spaces, Commun. Contemp. Math. 3 (2001), no. 1, 153-162. MR.1820017 (2002g:35192)

[3] Cazenave, T., Weissler, F. B., Some remarks on the nonlinear Schrödinger equation in the critical case, Nonlinear Semigroups, Partial Differential Equations, and Attractors (Washington, DC, 1987), 18-29, Lecture Notes in Math., 1394, Springer, Berlin, 1989. MR1021011 (91a:35149)

[4] Cazenave, T., Weissler, F. B., The Cauchy problem for the nonlinear Schrödinger equation in $H^{1}$, Manuscripta Math. 61 (1988), no. 4, 477-494. MR952091 (89j:35114)

[5] Cazenave, T., Weissler, F. B., The Cauchy problem for the critical nonlinear Schrödinger equation in $H^{s}$, Nonlinear Anal. 14 (1990), no. 10, 807-836. MR1055532 (91j:35252)

[6] Cazenave, T., Weissler, F. B., Asymptotically self-similar global solutions of the nonlinear Schrödinger and heat equations, Math. Z. 228 (1998), no. 1, 83-120. MR.1617975|(99d:35149)

[7] Christ, M., Colliander, J., Tao, T., A priori bounds and weak solutions for the nonlinear Schrödinger equation in Sobolev spaces of negative order, J. Funct. Anal. 254 (2008), no. 2, 368-395. MR2376575

[8] Ferreira, L. C. F., Villamizar-Roa, E. J., Self-similar solutions, uniqueness and long-time asymptotic behavior for semilinear heat equations, Differential Integral Equations 19 (2006), no. 12, 1349-1370. MR2279332 (2008c:35122)

[9] Kato, T., On nonlinear Schrödinger equations. II. $H^{s}$-solutions and unconditional wellposedness, J. Anal. Math. 67 (1995), 281-306. MR1383498(98a:35124a)

[10] Kato, T., Nonlinear Schrödinger equations, Schrödinger Operators (Sønderborg, 1988) , $218-$ 263, Lecture Notes in Phys., 345, Springer, Berlin, 1989. MR 1037322 (91d:35202)

[11] Ginibre, J., Velo, G., On a class of nonlinear Schrödinger equations. I. The Cauchy problem, general case, J. Funct. Anal. 32 (1979), no. 1, 1-32. MR533218(82c:35057) 
[12] Ginibre, J., Velo, G., On the global Cauchy problem for some nonlinear Schrödinger equations, Ann. Inst. H. Poincaré Anal. Non Linéaire 1 (1984), no. 4, 309-323. MR778977 (87a:35164)

[13] Ginibre, J., Velo, G., The global Cauchy problem for the nonlinear Schrödinger equation revisited, Ann. Inst. H. Poincaré Anal. Non Linéaire 2 (1985), no. 4, 309-327 MR801582 (87b:35150)

[14] Grünrock, A., Herr, S., Low regularity local well-posedness of the derivative nonlinear Schrödinger equation with periodic initial data, SIAM J. Math. Anal. 39 (2008), no. 6, 18901920. MR2390318

[15] Planchon, F., On the Cauchy problem in Besov spaces for a non-linear Schrödinger equation, Commun. Contemp. Math. 2 (2000), no. 2, 243-254. MR1759790 (2001e:35157)

[16] Stein, E. M., Weiss, G., Introduction to Fourier analysis on Euclidean spaces, Princeton Mathematical Series, no. 32, Princeton University Press, Princeton, N.J., 1971. MR0304972 (46:4102)

[17] Vargas, A., Vega, L., Global wellposedness for $1 D$ non-linear Schrödinger equation for data with an infinite $L^{2}$ norm, J. Math. Pures Appl. (9) 80 (2001), no. 10, 1029-1044. MR1876762 $(2002 \mathrm{j}: 35290)$

Departamento de Matemática, Universidade Federal de Pernambuco, 50740-540, RECifE, PE, BRAZIL

E-mail address: pablo@dmat.ufpe.br

Departamento de Matemática, Universidade Federal de Pernambuco, 50740-540, Recife, PE, BraziL

E-mail address: lcff@dmat.ufpe.br

Escuela de Matemáticas, Universidad Industrial de Santander, A.A. 678, BucaraMANGA, Colombia

E-mail address: jvillami@uis.edu.co 